Acta Crystallographica Section D

Biological

Crystallography

ISSN 0907-4449

Dominic J. B. Hunter, ${ }^{a}$ Rachel Macmaster, ${ }^{a}$ Aleksander W.

Roszak, ${ }^{a}$ Alan RiboldiTunnicliffe, ${ }^{a}$ Ian R. Griffiths ${ }^{b}$ and Andrew A. Freer ${ }^{\mathrm{a} *}$

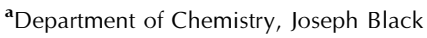
Building, University of Glasgow,

Glasgow G12 8QQ, Scotland, and ${ }^{\mathbf{b}}$ Applied Neurobiology Group, University of Glasgow, Veterinary School, Bearsden Road,

Glasgow G61 1QH, Scotland

Correspondence e-mail: andy@chem.gla.ac.uk

\title{
Structure of myelin P2 protein from equine spinal cord
}

Equine P2 protein has been isolated from horse spinal cord and its structure determined to $2.1 \AA$. Since equine myelin is a viable alternative to bovine tissue for large-scale preparations, characterization of the proteins from equine spinal cord myelin has been initiated. There is an unusually high amount of P2 protein in equine CNS myelin compared with other species. The structure was determined by molecular replacement and subsequently refined to an $R$ value of $0.187\left(R_{\text {free }}=\right.$ 0.233). The structure contains a molecule of the detergent LDAO and HEPES buffer in the binding cavity and is otherwise analogous to other cellular retinol-binding proteins.

\section{Introduction}

In our laboratory, equine tissue has replaced well characterized bovine tissue. Since most of our research involves spinal cord, there is an inherent risk of contracting variant Creutzfeldt-Jakob disease (the human form of bovine spongiform encephalopathy, BSE) if we were to continue using bovine tissue. As BSE presents problems in several countries, an alternate source of spinal cord is desirable: equine tissue provides the necessary amount for bulk experiments. Up until now very little if any work has been performed to characterize horse myelin. Although the myelin proteins are largely conserved across species, our initial investigations have shown that although similar to other well defined myelin proteins (e.g. bovine, human, rat, murine), equine myelin has some distinct differences. For example, our initial investigations into the major component of basic protein (Wood et al., 2002) shows a higher molecular weight of 18766 compared with 18501 and 18635 for human and bovine, respectively, owing to the increased number of amino acids in the equine protein: 172 compared with 170 for human and 169 for bovine. While careful isolation of the MBP isoforms by CM52 cationexchange chromatography shows the same panoply of charged isoforms, there was one unexpected result: the large amount of $\mathrm{P} 2$ protein present in equine CNS tissue.

Myelin P2 protein is normally found as a small $(14.8 \mathrm{kDa})$ component of the peripheral nervous system (PNS), making up some $2-15 \%$ of the total PNS protein (Kadlubowski et al., 1984). It is very probably localized to the cytoplasmic face of the Schwann cell membrane (Weize \& Brostoff, 1985), where it associates with the membrane. Its exact function is unclear, although it is obvious from experimental evidence (Uyemura et al., 1984) and its relation to other hydrophobic ligandbinding proteins that this function involves the binding of lipophiles, thereby suggesting a role in lipid transport and storage in myelinating Schwann cells (Uyemura et al., 1984). (The paradox, however, is that most of the major lipids found
Received 7 February 2005

Accepted 3 May 2005

PDB Reference: equine P2 myelin, 1 yiv, r1 yivsf.
(C) 2005 International Union of Crystallography Printed in Denmark - all rights reserved 
in myelin, e.g. cholesterol and phosphatidylcholine, are very much larger than the binding pocket in $\mathrm{P} 2$ and hence its role may be to shuttle the lipid components.)

In common with many myelin proteins, injection of purified P2 protein (Kadlubowski \& Hughes, 1979) or neuritogenic peptides derived from it (Uyemura et al., 1982) into experimental animals results in an experimental allergic neuritis (EAN). In the case of P2, an animal model for Guillain-Barré syndrome, a demyelinating disease of human PNS, is produced. Interestingly, in horses there is a condition known as neuritis of the cauda equina (NCE), which leads to paralysis of the tail, bladder and rectum. Although the aetiology of the condition is unknown, the paralysis of the trigeminal and facial nerves show similarities to Guillain-Barré syndrome in man and experimental allergic neuritis (EAN) in animals (Kadlubowski \& Ingram, 1981; Greenwood et al., 1973).

On the basis of both sequence and three-dimensional structure, myelin P2 protein is closely related to cellular lipophilic transport proteins such as cellular retinol-binding proteins (CRBP, CRBP II; Bashor et al., 1973; Li et al., 1986), heart fatty-acid-binding protein (Heuckeroth et al., 1987), intestinal fatty-acid-binding protein (Sacchettini et al., 1988) and the cellular retinoic acid-binding proteins (CRABP, CRABP II; Sundelin et al., 1985; Bailey \& Siu, 1988; Kitamoto et al., 1988). The structures of several of these proteins are known, including that of bovine myelin $\mathrm{P} 2$ protein (Jones et al., 1988; Cowan et al., 1993), all of which show the same tenstranded antiparallel $\beta$-barrel closed at one end by a helixturn-helix motif in the large loop between strands 1 and 2 of the barrel. The other inter-strand loops are short. The serum retinol-binding protein family (serum RBP, $\beta$-lactoglobulin etc.) differs from the above, having an eight-stranded antiparallel $\beta$-barrel with $\mathrm{N}$ - and $\mathrm{C}$-terminal extensions.

In this paper, we describe the purification and structure determination of $\mathrm{P} 2$ protein from horse central nervous system (CNS) tissue to high resolution. We routinely extract P2 from our detergent-solubilized fractions of MBP and PLP and, as an added experiment, decided to keep the solubilizing detergent LDAO (lauryl dimethylamine- $N$-oxide) present at its CMC $(0.1 \%)$ throughout the purification and crystallization protocol, almost treating it like a membrane protein in many respects (although it is not), just to see if the copious lipid present would be incorporated into the active site or liganded to the exterior surface of the protein. $\mathrm{P} 2$ can also be easily extracted from equine CNS tissue by simply using buffer and $200 \mathrm{mM} \mathrm{NaCl}$ and gently agitating by homogenizing or gentle sonication. Visual inspection of the remaining solution by SDS-PAGE indicates that P2 extraction is almost complete. Since there is a relatively large amount of $\mathrm{P} 2$ in equine myelin (between 5 and $10 \%$ of the total myelin proteins), this initial pre-solubilization is a useful clean-up step prior to removal of MBP and PLP proteins.

\section{Materials and methods}

All materials used in the purification were of reagent grade or higher. Lauryl dimethylamine- $N$-oxide (LDAO) was from
Fluka, Dorset, England. Cation-exchange chromatography used Whatman CM52 cellulose and Superdex 75 (Amersham Bioscience, UK) was used for gel filtration.

\subsection{Purification of $\mathbf{P} 2$ protein}

Myelin was purified from horse spinal cord by the method of Norton \& Poduslo (1973). P2 protein was extracted from myelin using a low concentration of the detergent LDAO and purified by chromatography on CM52 cellulose. Briefly, myelin was suspended at $\sim 2 \mathrm{mg} \mathrm{ml}^{-1}$ protein in $10 \mathrm{mM}$ PIPES pH 6.8, $5 \mathrm{~m} M$ EDTA, $200 \mathrm{~m} M \mathrm{KCl}, 0.1 \%(v / v)$ LDAO by gentle stirring. The suspension was sonicated for $2 \mathrm{~min}$ (bath sonicator) and then stirred gently for $20 \mathrm{~min}$. Following

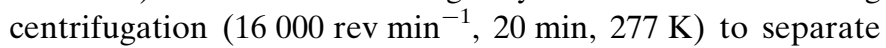
the extract, the residue was re-extracted a further three times. The soluble extracts were pooled and dialysed overnight against $10 \mathrm{~m} M$ Tricine $\mathrm{pH} 8.9,1 \mathrm{~m} M$ EDTA, $0.1 \%(v / v)$ LDAO and centrifuged [18 $000 \mathrm{rev} \mathrm{min}^{-1}, 30 \mathrm{~min}, 277 \mathrm{~K}$ ] to remove precipitated lipid and contaminating cyclic nucleotide 3'-phosphatase (CNPase). The clarified extract was concentrated (Amicon Ultrafiltration, YM10 membrane from Millipore UK Ltd) and applied onto a $1 \times 20 \mathrm{~cm}$ column of CM 52 cellulose equilibrated with $10 \mathrm{~m} M$ PIPES $\mathrm{pH}$ 6.8, $1 \mathrm{~m} M$ EDTA, 0.1\% LDAO. After washing, the bound proteins, including P2, were eluted with a linear $0-0.5 \mathrm{M}$ gradient of $1 M \mathrm{NaCl}$ in equilibration buffer. Fractions containing P2, as judged by SDS-PAGE, were pooled, concentrated to $\sim 1 \mathrm{ml}$ and applied onto a Superdex $75(1.6 \times 50 \mathrm{~cm}$ column $)$ in the above equilibration buffer to remove minor contaminants. The S-75 eluate was concentrated, typically to $5 \mathrm{mg} \mathrm{ml}^{-1}$ protein, and stored at $253 \mathrm{~K}$.

Pure P2 protein was subject to electrospray mass spectrometry following inline HPLC to remove buffer components. From the deconvoluted ion series, the major sample component gave a weight of $14761 \mathrm{Da}$.

\subsection{Crystallization, data collection, structure solution and refinement}

Purified P2 protein formed crystals in several of the Hampton Research A screen solutions. Crystals grown using sitting-drop vapour diffusion in optimized condition No. 41 (0.1 $M$ HEPES pH 5.6, 20\% PEG 4000, 15\% 2-propanol) diffracted well. Data were collected to better than $2.1 \AA$ at stations 9.6 and 14.1 at SRS, Daresbury on a crystal form with space group $P 3_{2} 2_{1}$ and unit-cell parameters $a=b=45.9$, $c=125.8 \AA, \gamma=120^{\circ}$ at $100 \mathrm{~K}$ using $10 \%$ glycerol as cryoprotectant. Data were reduced using DENZO/SCALEPACK (Otwinowski \& Minor, 1997); subsequent data processing used the CCP4 suite. Interestingly, we frequently obtained drops in which two crystal forms had grown simultaneously: the form used in this analysis, shaped like thick prisms, and also fine long needle-shaped crystals. Synchrotron data were collected from several of these needle-shaped crystals, but they only diffracted to about $4.5 \AA$ in monoclinic space group $C 2$.

The structure was solved by molecular replacement using AMoRe (Navaza, 1994). P2 bovine myelin protein stripped of 

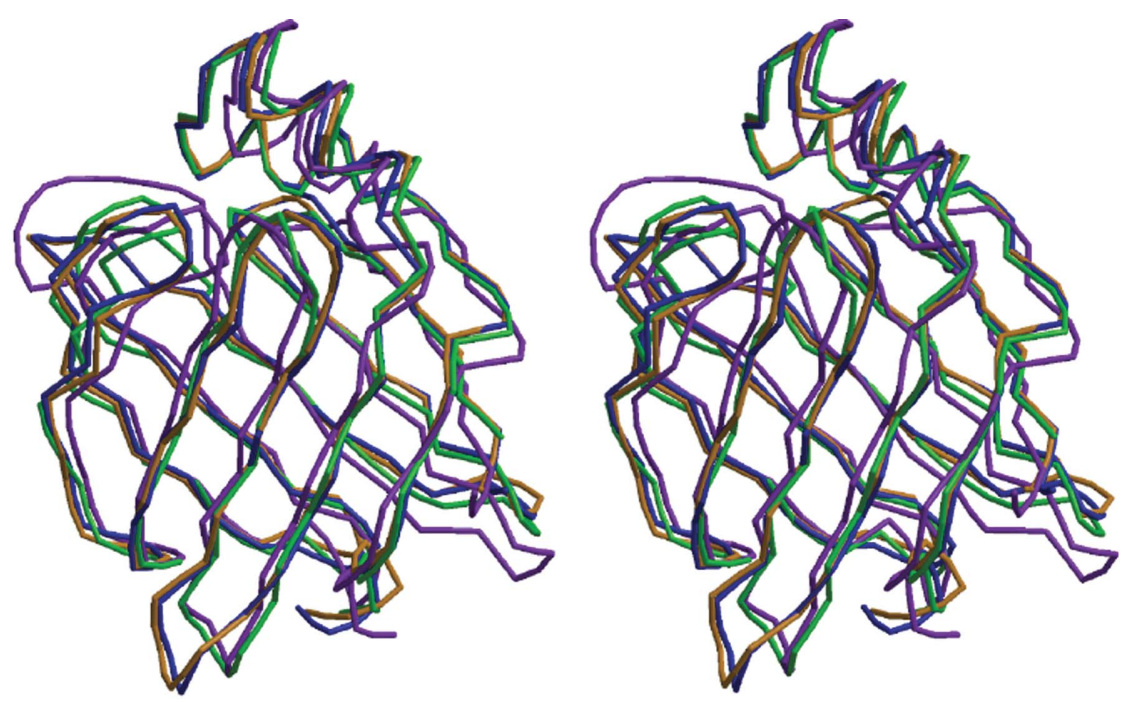

Figure 1

Stereo overlay of equine (dark blue), rat (green), bovine (orange) and human (purple) P2 structures.

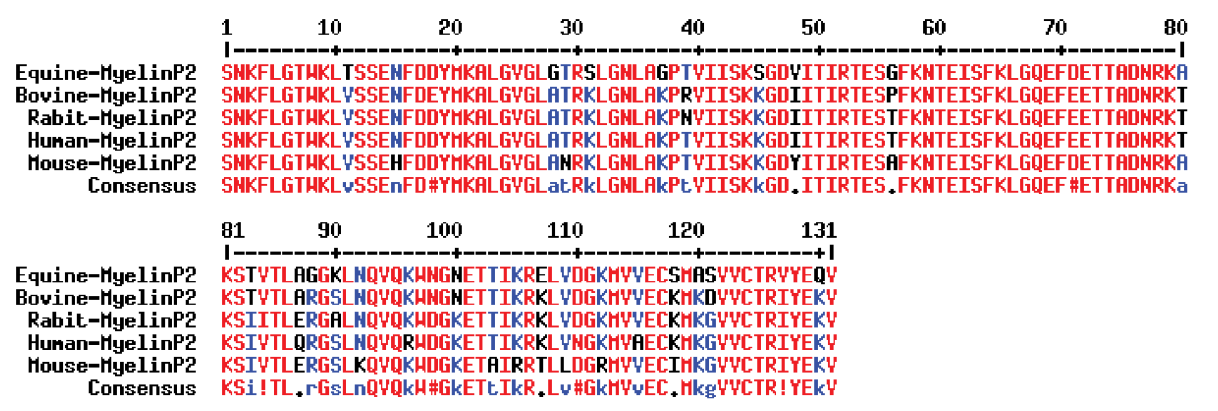

Figure 2

Sequence alignment of equine, bovine, rabbit, human and mouse myelin P2 proteins.
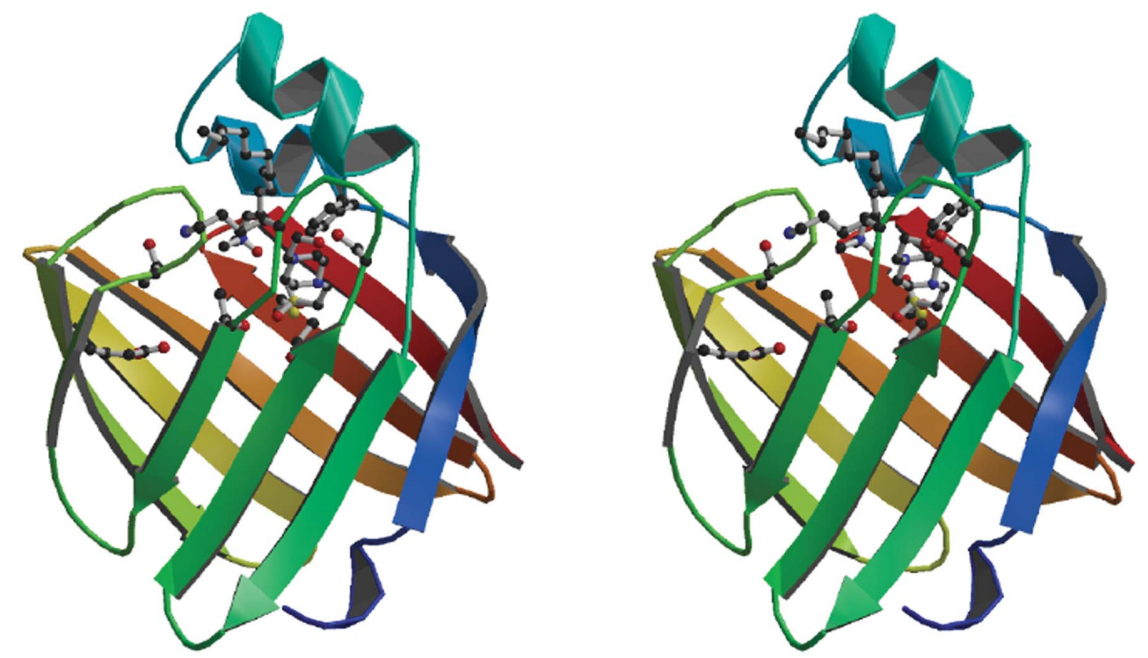

Figure 3

View of binding pocket with HEPES and LDAO.

water molecules (PDB code 1pmp) was used as the initial search molecule. Before refinement, $5 \%$ of the observed reflections were flagged for calculation of the free $R$ factor, $R_{\text {free. }}$ The structure was initially refined using $R E F M A C$ (Murshudov et al., 1997) and the P2 structure was manually built into the resulting electron-density map using QUANTA. As no sequence is available for equine $\mathrm{P} 2$, the assignment of amino acids was based on sequence alignment of four other myelin P2 sequences (Fig. 2). Side chains were added when electron density permitted. Several iterative rounds of model building and refinement allowed over $95 \%$ of the protein model to be built. Subsequent rounds of model building and refinement (again using REFMAC) involved TLS and restrained refinement (Winn et al., 2001). The final model contains 1016 protein atoms, 102 water molecules, a HEPES molecule and a detergent LDAO molecule. The final $R$ factor is 0.187 and $R_{\text {free }}$ is 0.233 . Table 1 outlines the refinement parameters. (space group $P 3_{2} 2_{1}$ was confirmed over the symorphic $P 3_{1} 2_{1}$ by initial trial refinements in each space group).

\section{Results and discussion}

The overall structure is homologous to other P2 and CRBP structures determined to date, comprising a single domain consisting of a ten-stranded upand-down $\beta$-barrel with two orthogonal $\beta$-sheets. The top sheet consists of strands 1-4 and the bottom sheet of strands $5-10$; strand 1 is shared by both sheets, with a classic $\beta$-bulge (Chan et $a l ., 1993)$ seen at residue 11 . The centre of the barrel is closed at one end by two short $\alpha$-helices. Residues Met119 and Trp97 and a salt bridge between OD1 and OD2 on Asp76 and NE and NH2 on Arg78 on neighbouring loops also close the opening. The entrance at the other end of the barrel is restricted by a number of conserved hydrophobic residues pointing into the barrel. These include Ser1, Lys44, Ile49, Leu66 and Leu91. Access to the interior of the barrel is gained by a small orifice formed between the second helix and the second hairpin turn involving Thr29, Leu32, Phe57 and Lys58. The opening is controlled by the conformation of Phe57 (Cowan et al., 1993). Fig. 1 illustrates the similarity in secondary-structure topology by overlaying the current equine structure (dark blue) with three other CRBP structures (rat, green, PDB code 1 crb; bovine, orange, 1pmp; human, purple, 1cbq). The equine, rat and bovine structures have a 
Table 1

Refinement parameters.

\begin{tabular}{ll}
\hline Space group & $P 3_{2} 2_{1}$ \\
Unit-cell parameters $\left(\AA{ }^{\circ}\right)$ & $a=45.846, b=45.846, c=125.778$, \\
& $\alpha=90.0, \beta=90.0, \gamma=120.0$ \\
Resolution limits & $41.89-2.10$ \\
No. of unique reflections & 8255 \\
Completeness $(\%)$ (high-resolution shell) & $91.51(67.93)$ \\
$R_{\text {work }} / R_{\text {free }}(\%)$ & $18.6 / 23.3$ \\
R.m.s. bond lengths $(\AA)$ & 0.018 \\
R.m.s. bond angles $\left({ }^{\circ}\right)$ & 1.831 \\
No. of protein residues & 131 \\
No. of water molecules & 102 \\
No. of hetero molecules & LDAO, HEPES \\
Average $B$ factor $\left(\AA^{2}\right)$ & 43.22 \\
Percentage of residues in Ramachandran & plot regions \\
$\quad$ Most favoured & 92.4 \\
Additional allowed & 5.1 \\
Generously allowed & 1.7 \\
\hline
\end{tabular}

near-identical match, with the human structure showing slight deviations in the loop between $\beta$-strands 5 and 6 (at the open end of the barrel) and the small loop between strands 8 and 9 .

There are two significant mutations in equine P2 when compared with other myelin P2 sequences (Fig. 2): Arg88 and Lys120 are both mutated to Ala. This would deplete the overall positive charge on the molecule by 2 when compared with other myelin P2s. A lack of density in these regions suggested other assignments to be inappropriate. In several cellular retinol-binding proteins (CRBPs), Arg88 is replaced with a glycine. Since there is no sequence information for equine $\mathrm{P} 2$, the side-chain assignments were made solely from electron-density modelling, taking cognisance of sequence information from other species. The electron density for the structure was very good and these different side-chain residues were only confirmed after careful refinement. Other minor mutations were Val for Ile48 and Ala for Thr80 and Ser90. Fig. 2 shows the sequence alignment of equine, bovine, rabbit, human and mouse myelin $\mathrm{P} 2$, illustrating the high degree of conservation between species. Electrospray mass spectrometry gave a mass of 14761 , while the calculated mass from the sequence gives 14288. However, when the contribution from the HEPES and LDAO molecules that are found in the cavity is added in, this brings the weight to $14756 \mathrm{Da}$, which gives further confidence to the assigned residues.

\subsection{Binding site and ligands}

Two molecules have been found in the binding site: the detergent LDAO and HEPES buffer, both sequestered from the crystallization conditions (Fig. 3). The HEPES buffer molecule is at the hydrophilic end of the compartment. Each of the $\mathrm{O}$ atoms on the sulfonyl head group makes contact with different moieties: $\mathrm{O}(1)$ to water $61, \mathrm{O}(2)$ to Cys117 and $\mathrm{O}(3)$ to the oxygen at the head of the detergent, LDAO. Interestingly, the hydroxyl group of the buffer points inwardly rather than in an extended conformation to allow favourable contacts with Ser55 OG and the carbonyl O atom of Lys58. With the close proximity of the adjacent hydrophobic region of LDAO this $\mathrm{O}-\mathrm{H}$ rotation makes sense. There are nine water molecules that appear to form a halo-type motif around the HEPES group. The positioning of a number of water molecules (usually between five and nine) at this end of the barrel seems to be a common feature of P2 and CRBP structures.

The tail of the detergent makes favourable hydrophobic interactions with the residues present in the opening at the second hairpin turn, while the head group makes interactions with HEPES $\mathrm{O}(3)$ and a number of water molecules in the 'halo'. There is also a hydrophobic interaction (3.78 $\AA$ ) between $\mathrm{C}(8)$ of HEPES and $\mathrm{C}(4)$ of LDAO. The above arrangement is not dissimilar to that found in the insect fattyacid-binding protein isolated from Manduca sexta (Benning et al., 1992), where a sulfate ion derived from crystallization in ammonium sulfate is also seen at the hydrophilic end of the barrel along with the fatty acid, palmitic acid. Initially, we tried to model a retinol molecule into this site since the aromatic ring was clearly defined in the initial density. However, there was no connective density from this proposed head group to the unsaturated chain (of the subsequent LDAO).

\section{Conclusion}

Structurally, equine $\mathrm{P} 2$ is practically identical to other $\mathrm{P} 2$ and CRBP structures, even though the equine myelin was derived from CNS rather than PNS tissue. Careful examination of the residual density from difference maps showed no evidence of lipid or other molecules associated with the exterior of the protein despite careful extraction, purification and crystallization under detergent conditions. This is in contrast to the lipid-bound form of bovine $\mathrm{P} 2$ crystallized by Sedzik et al. (2003). SDS-PAGE clearly shows lipid being carried forward in our purification steps and probably into crystallization. It may be that the second form of poorly diffracting rod-shaped crystals does have associated lipid and hence the heterogeneity of the system prevents the growth of better diffracting crystals. The presence of HEPES and LDAO molecules in the binding pocket is not surprising since these molecules, derived from purification and crystallization procedures, fit the requirements of the binding pocket: a charged entity at the hydrophilic end and a hydrophobic tail pointing towards the opening at the second hairpin turn.

We are grateful for myelin preparation (Mr D. Kirkham, Glasgow University, Veterinary School) and for funding (Medical Research Council).

\section{References}

Bailey, J. S. \& Siu, C. H. (1988). J. Biol. Chem. 263, 9326-9332.

Bashor, M. M., Toft, D. O. \& Chytil, F. (1973). Proc. Natl Acad. Sci. $U S A$, 70, 3483-3487.

Benning, M. M., Smith, A. F., Wells, M. A. \& Holden, H. M. (1992). J. Mol Biol. 228, 208-219.

Chan, E. A. W., Hutchinson, E. G., Harris, D. \& Thornton, J. M (1993). Protein Sci. 2, 1574-1590.

Cowan, S. W., Newcomer, M. E. \& Jones, T. A. (1993). J. Mol. Biol. 230, 1225-1246.

Greenwood, A. G., Barker, J. \& McLeich, I. (1973). Equine Vet. J. 5, 111-115. 
Heuckeroth, R. O., Birkenmeier, E. H., Levin, M. S. \& Gordon, J. I. (1987). J. Biol. Chem. 262, 9709-9717.

Jones, T. A., Bergfors, T., Sedzik, J. \& Unge, T. (1988). EMBO J. 7, 1597-1604.

Kadlubowski, M. \& Hughes, R. A. C. (1979). Nature (London), 277, 140-141.

Kadlubowski, M., Hughes, R. A. C. \& Gregson, N. A. (1984). J. Neurochem. 42, 123-129.

Kadlubowski, M. \& Ingram, P. L. (1981). Nature (London), 293, 299 300.

Kitamoto, T., Momoi, T. \& Momoi, M. (1988). Biochem. Biophys. Res. Commun. 157, 1302-1308.

Li, E., Demmer, L. A., Sweetser, D. A., Ong, D. E. \& Gordon, J. I. (1986). Proc. Natl Acad. Sci. USA, 83, 5779-5783.

Murshudov, G. N., Vagin, A. A. \& Dodson, E. J. (1997). Acta Cryst. D53, 240-255.

Navaza, J. (1994). Acta Cryst. A50, 157-163.

Norton, W. T. \& Poduslo, S. E. (1973). J. Neurochem. 21, 749-758.
Otwinowski, Z. \& Minor, W. (1997). Methods Enzymol. 276, 307-326. Sacchettini, J. C., Gordon, J. I. \& Banaszak, L. J. (1988). J. Biol. Chem. 263, 5815-5819.

Sedzik, J., Carlone, G., Fasano, A., Liuzzi, G. M. \& Riccio, P. (2003). J. Struct. Biol. 142, 292-300.

Sundelin, J., Das, S. R., Eriksson, U., Rask, L \& Peterson, P. A. (1985). J. Biol. Chem. 260, 6494-6499.

Uyemura, K., Suzuki, M., Kitamura, K., Horie, K., Ogawa, Y., Matsuyama, H., Nozaki, S. \& Muramatsu, I. (1982). J. Neurochem. 39, 895-898.

Uyemura, K., Yoshimura, K., Suzuki, M. \& Kitamura, K. (1984). Neurochem. Res. 9, 1509-1514.

Weize, M. J. \& Brostoff, S. W. (1985). Res. Methods Neurochem. 6, 263-302.

Winn, M. D., Isupov, M. N. \& Murshudov, G. N. (2001). Acta Cryst. D57, 122-133.

Wood, D., She, Y., Freer, A., Harauz, G. \& Moscarello, M. (2002). Arch. Biochem. Biophys. 405, 137-146. 